\title{
On Asymptotic Behaviors of Exponential Hedging in the Basis-Risk Model
}

\author{
Kazuhiro Takino \\ Faculty of Commerce, Nagoya University of Commerce and Business, Nisshin, Japan \\ Email: takino@nucba.ac.jp
}

Received 17 April 2015; accepted 24 May 2015; published 27 May 2015

Copyright (C) 2015 by author and Scientific Research Publishing Inc.

This work is licensed under the Creative Commons Attribution International License (CC BY). http://creativecommons.org/licenses/by/4.0/

(c) (i) Open Access

\begin{abstract}
In this article, we consider the exponential hedging and the mean-variance hedging in the basis-risk model. We construct hedging strategies for multiple units of claim and calculate hedging errors. We then observe how the hedge error risk increases when the investor raises trading volumes of the claim. Under our definition of the hedge error risk amount, the risk increases in a linear way, according to the claim volume for the mean-variance hedging. As to the exponential hedging, it does not, i.e., nonlinear increment. The hedging error for the exponential hedging, however, tends to have the same properties to the mean-variance hedging when either risk-averse parameter or claim volume goes to zero. We numerically demonstrate this fact. Our numerical demonstration with the results of the previous researches verifies that the indifference price converges to the mean-variance hedging cost when the claim volume goes to zero under the basisrisk model.
\end{abstract}

\section{Keywords}

Basis-Risk Model, Exponential Hedging, Indifference Pricing, Mean-Variance Hedging

\section{Introduction}

In this article, we consider hedging problems for the European-type contingent claim taking into account the position of the claim on the basis-risk model. We consider both exponential hedging and mean-variance hedging for multiple units of claim. We only consider the seller's problems for convenience, the position, thus implying the sold amount, which is also called claim volume in this study. In the previous studies, the exponential hedging problems and the mean-variance hedging problems have been solved for one unit of claim. However, in practice, many financial institutions trade great numbers of derivatives. If they want to maintain the solvency margin or the capital adequacy, they should manage or control their risks taking into account their positions. Hence, it is 
needed to consider the hedging problems for multiple units of the claim.

The basis-risk model is a typical example of the incomplete market model, which includes the model that the underlying asset of the contingent claim is not traded in the financial market. The pricing models for the weather derivative or the derivative written on the market index are recognized as one of the basis-risk models for instance. In the complete market (e.g., Black-Scholes model), any contingent claims are perfectly replicated with traded assets, and this simultaneously gives the price of the claim. On the other hand, the value of the claim is not surly attained with traded assets in the incomplete market setting. This means that the seller of the claim is exposed to have the hedge error risk, so she/he wants to control it with her/his preference. The exponential hedging and the mean-variance hedging have independently developed in the context of finding the optimal hedging strategy for the contingent claim in the incomplete market model. The significant difference of the both approaches is whether it includes the risk preference of the market participant or not. The exponential hedging reflects the investor's attitude for the risk since it is based on the utility maximization with the exponential utility. The exponential hedging also has been developed in context of the utility indifference pricing with the exponential utility such as [1]-[8]. We use the indifference price as the initial cost of the exponential hedging. The exponential hedging approach is usually formulated to maximize the expected utility for the amounts of which the hedge portfolio exceeds the claim payoff. The mean-variance hedging, on the other hand, is a hedging criterion to minimize the hedge error measured by $\mathcal{L}_{2}$-norm, and does not take into account the investor's preference for the risk. This problem is solved by using the projection in the Hilbert space (see [9] for more details).

These methods do not only provide the optimal portfolio strategy, but also lead the pricing rule including the selection of the equivalent martingale measure. Davis [10], Frittelli [11] and Delbaen et al. [1] respectively developed the dual problem of the primal exponential hedging problem, and showed that the minimal martingale measure is given by their duality theories. Adding their contributions, the exponential hedging has been sophisticated as the robust utility maximization framework such as [8]. The mean-variance hedging determines the equivalent martingale measure called variance-optimal martingale measure under which the price of the claim is given by the expected value of the discount payoff, i.e., no arbitrage price.

It is recalled that we consider the hedging problems for multiple units of claim. We evaluate the hedge error risk with the squared root of the expectation of the quadratic hedge error (i.e., $\mathcal{L}_{2}$-norm) which is also the objective function of the mean-variance hedging. It is shown that the hedge error risk for the mean-variance hedging then changes in a linear way with respect to the claim volume in this paper. The case of the exponential hedging, however, does not hold in general. The hedge error risk of the exponential hedging climbs in a nonlinear way with respect to the claim volume as our numerical results show. We call this property nonlinear increment; we demonstrate this characteristic in numerical scheme.

At this point, the asymptotic behaviors for both exponential hedging and utility indifference price have been considered in previous literatures. Ilhan et al. [4] summarized that the utility indifference price converges to the no arbitrage price with the minimal martingale measure when either risk-averse coefficient or claim volume goes to zero. This fact is shown in this article too. Mania and Schweizer [12] showed that the exponential hedging strategy with the utility indifference price converges to the strategy of the mean-variance hedging when the risk-averse coefficient goes to zero. Therefore, combining Ilhan et al. [4], Mania and Schwiezer [12] identified that the utility indifference price converges to the mean-variance hedging cost when the risk-averse coefficient closes to zero. In fact, the variance-optimal martingale measure which is given in the mean-variance hedging coincides with the minimal martingale measure for the basis-risk model such as [13] [14]. On the other hand, Ilhan et al. [4] described that the indifference price converges to the superhedging price when either risk-averse coefficient or claim volume goes to infinity. Also, from numerical examples of [5], one can observe that the exponential hedging takes the distribution of the hedge error/performance as same as the superhedging for the large risk-averse coefficient.

Reviewing previous researches makes us be aware that it has never verified the implication about the convergence goal of the indifference price and the exponential hedging when the claim volume goes to zero, for instance. This study considers and investigates that how the indifference price and the exponential hedging converge when the claim volume goes to zero. We implement the exponential hedging and observe its hedge error closing the claim volume zero. We then find that the hedge error of this hedging approach has linearity with respect to the claim volume for small claim volume. This property is also characterized for the mean-variance hedging as mentioned in the above. That is, the exponential hedging tends to have the same behavior to the one of the mean-variance hedging when the claim volume goes to zero. From our demonstration with the review of Ilhan et al. [4], therefore it is interpreted that for the basis-risk model the utility indifference price converges to 
the mean-variance hedging cost when the claim volume goes to zero.

The rest of the paper is organized as follows: in Section 2, we set up the financial market model. We especially consider the basis-risk model. In Section 3, we solve the mean-variance hedging problem for the multiple units of claim. Also, we show the linear increment of the hedge error risk for the mean-variance hedging strategy. In Section 4, we construct the exponential hedging with the utility indifference price for multiple units of claim. In particular, we derive the exponential hedging strategy by asymptotic scheme. In Section 5, we implement the exponential hedging and numerically demonstrate behaviors of hedge error amounts for both hedging strategies. Finally, we conclude this study in Section 6.

\section{Model}

\subsection{Financial Market Model}

We consider the basis-risk model (or non-traded asset model). That is, there are one risky asset $S$ (typically the stock), one risk-free asset $B$ (typically the bank account) with zero risk-free rate and one state level $Y$ which is supposed to be not traded in the financial market. For instance, as to the weather derivative case, $Y$ corresponds to a weather index such as the average temperature. Let us set the value process for above instruments. The uncertainty in this market is characterized by a probability space $(\Omega, \mathcal{F}, P)$. We then introduce a two-dimensional standard Brownian motion denoted by $W=\left(W_{1}, W^{\perp}\right)$ on $\left(\Omega, \mathcal{F}, P ; \mathcal{F}_{t}\right)$, where $F_{t}$ is the filtration generated by $(W(t) ; 0 \leq s \leq t)$ and satisfies the usual conditions.

The value process of the risk-free asset $B$ is

$$
\mathrm{d} B(t)=0
$$

with $B(0)=1$ and risk-free rate is 0 . The stock price process $S$ and the state level $Y$ are supposed to be driven by

$$
\begin{array}{r}
\mathrm{d} S(t)=S(t)\left\{\mu_{1} \mathrm{~d} t+\sigma_{1} \mathrm{~d} W_{1}(t)\right\} \\
\mathrm{d} Y(t)=Y(t)\left\{\mu_{2} \mathrm{~d} t+\sigma_{2} \mathrm{~d} W_{2}(t)\right\}
\end{array}
$$

for $0 \leq t \leq T$, where $W_{2}:=\rho W_{1}+\sqrt{1-\rho^{2}} W^{\perp} \quad(-1<\rho<1), \quad \mu_{i}$ and $\sigma_{i} \quad(i=1,2)$ are constants.

We would price a European-type claim whose payoff function is denoted by $H(T):=H(T, Y(T))$ at maturity $T$. In the numerical simulation, we consider the put option as an example. This allows us to use an asymptotic expansion introduced by Monoyios [10]. We assume $H \in \mathcal{L}_{2}(P)$, where $\mathcal{L}_{2}(P)$ is a space of square integrable random variables, i.e.,

$$
\mathcal{L}_{2}(P)=\left\{X: E\left|X^{2}\right|<\infty\right\}
$$

We use European put option in the numerical example.

The hedging strategies are constructed by the self-financing rule. That is, the hedge portfolio value $X(t)$ $(0 \leq t \leq T)$ is driven by

$$
\mathrm{d} X(t)=\kappa(t) \mathrm{d} S(t), X(0)=x
$$

with the hedging strategy $\kappa$ which is the amount held in the stock $S . X(0)$ corresponds the initial hedging cost, it is assigned to the utility indifference price in the exponential hedging as explained in the following section. Now we give the mathematical condition of $\kappa$, i.e., admissible policy.

Definition 2.1. (Admissible) The portfolio strategy $\kappa$ is admissible if it satisfies

$$
E\left[\int_{0}^{T} \kappa^{2}(t) S^{2}(t) \mathrm{d} t\right]<\infty
$$

Therefore, we denote by $A$ the set of all admissible policies $\kappa$.

Because of the incomplete market model both strategies are exposed to have hedging error. In this work, the hedging errors $R^{(k)}$ for two hedging strategies are supposed to be measured by

$$
R^{(k)}=\left(E[k H(T)-X(T)]^{2}\right)^{1 / 2} .
$$




\subsection{Equivalent Martingale Measure}

For two-dimensional predictable process $\lambda=\left(\lambda_{1}, \lambda_{2}\right)$ we introduce

$$
Z(t)=\mathcal{E}(-\lambda \cdot W):=\exp \left(-\frac{1}{2} \int_{0}^{t}\left(\lambda_{1}(s)^{2}+\lambda_{2}(s)^{2}\right) \mathrm{d} s-\int_{0}^{t}\left(\lambda_{1}(s) \mathrm{d} W_{1}(s)+\lambda_{2}(s) \mathrm{d} W^{\perp}(s)\right)\right) .
$$

We assume that $\theta:=\lambda_{1}(t) \equiv \mu_{1} / \sigma_{1}$ and $\lambda=\left(\lambda_{1}, \lambda_{2}\right)$ satisfies Novikov condition

$$
E\left[\mathrm{e}^{\frac{1}{2} \int_{0}^{t}\left(\lambda_{1}(s)^{2}+\lambda_{2}(s)^{2}\right) \mathrm{d} s}\right]<\infty
$$

Then $Z$ is a martingale under $P$. $Z$ is a solution of

$$
\mathrm{d} Z(t)=-Z(t)\left(\lambda_{1}(s) \mathrm{d} W_{1}(s)+\lambda_{2}(s) \mathrm{d} W^{\perp}(s)\right), Z(0)=1 .
$$

Defining an equivalent probability measure $P^{\lambda}$ by

$$
\frac{\mathrm{d} P^{\lambda}}{\mathrm{d} P}=Z(T)
$$

then $P^{\lambda}$ is an equivalent martingale measure. Under $P^{\lambda}$ measure, the risky asset price discounted with the risk-free asset becomes a martingale. Set $\lambda=(\theta, 0)$ then yields the minimal martingale measure denoted by $Q$. The density process of $Q$ is also given by $Z(t)=\mathcal{E}\left(-\theta W_{1}\right)$. Define $\tilde{W}=\left(\tilde{W}_{1}, \tilde{W}^{\perp}\right)$ by

$$
\tilde{W}_{1}(t)=W_{1}(t)+\int_{0}^{t} \theta \mathrm{d} s, \tilde{W}^{\perp}(t)=W^{\perp}(t),
$$

then, from the Girsanov's theorem, $\tilde{W}$ is two-dimensional Brownian motion under $Q$.

\section{Mean-Variance Hedging}

In the present section, we consider the mean-variance hedging strategy for multiple units of claim. The result argued in this section is a basis for the main theorem. The purpose of the mean-variance hedging is to find a hedge portfolio strategy $\kappa^{(k)}(t) \in \mathcal{A} \quad(0 \leq t \leq T)$ with the initial cost $C^{(k)}$ (constant) to minimize the hedge error with $\mathcal{L}_{2}$-norm, i.e., to minimize $R^{(k)}$ defined in (2.1). Define so-called gain process $G^{(k)}$ by

$$
G^{(k)}(t)=\int_{0}^{t} \kappa^{(k)}(s) \mathrm{d} S(s),
$$

then the value process of the hedge portfolio is represented by

$$
X(t)=C^{(k)}+G^{(k)}(t)
$$

since the initial hedging cost $X(0)$ is $C^{(k)}$. Our purpose is therefore to find $\kappa^{(k)}$ such that

$$
\min _{\kappa^{(k)} \in \mathcal{A}} R^{(k)}=\min _{\kappa^{(k)} \in \mathcal{A}}\left(E\left[k H(T)-C^{(k)}-G^{(k)}(T)\right]^{2}\right)^{\frac{1}{2}} \text {. }
$$

In the rest of the section, we construct the mean-variance hedging strategy for multiple units of claim (i.e., $k H$ ) and evaluate its hedging error (3.1).

\subsection{Variance-Optimal Martingale Measure}

The mean-variance hedging strategy is constructed with Galtchouk-Kunita-Watanabe decomposition of the claim $H$ under so-called Variance Optimal Martingale Measure (VOMM). We denote VOMM by $P^{*}$. In particular, the initial hedging $\operatorname{cost}^{1}$ is given by the expected value of the discounted payoff of $H$ under VOMM. We would like to recommend the reader to refer [9] [15] more detail explanations for the mean-variance hedging. We thus first need to specify VOMM $P^{*}$. We define the VOMM according to [15].

Definition 3.1. (Variance-Optimal Martingale Measure: VOMM) The equivalent martingale measure $P^{\lambda}$ is the variance-optimal martingale measure if it solves to

\footnotetext{
${ }^{1}$ This value is also called Approximation Price of $H$ in [9] [16].
} 


$$
\inf _{P^{\lambda} \in \mathcal{M}_{e}} E\left[\frac{\mathrm{d} P^{\lambda}}{\mathrm{d} P}\right]^{2} .
$$

It is easy to find the VOMM for our basis-risk model.

Proposition 3.1. (Variance-Optimal Martingale Measure) The variance-optimal martingale measure $P^{*}$ is given by

$$
\frac{\mathrm{d} P^{*}}{\mathrm{~d} P}=Z^{*}(t):=\mathcal{E}\left(-\theta W_{1}\right)
$$

in our financial market model introduced in the previous section.

Proof. Under the real world measure $P$, the discount risky asset price $\hat{S}$ is represented by using the martingale term $M$ and the finite variation $A$

$$
\hat{S}(t)=\hat{S}(0)+M(t)+A(t)
$$

where $M(t)=\int_{0}^{t} \sigma_{1} X(s) \mathrm{d} W_{1}(s)$ and $A(t)=\int_{0}^{t} \eta(s) \mathrm{d}\langle M\rangle(s)$ with $\eta(t)=\theta / \sigma_{1} X(t)$. Then the mean-variance tradeoff process $J$ defined by

$$
J(t)=\int_{0}^{t} \eta(s)^{2} \mathrm{~d}\langle M\rangle(s)
$$

is then deterministic. Therefore, Lemma 4.7 in [9] completes the proof.

Q.E.D.

From Proposition 3.1, $Z^{*}$ solves to

$$
\mathrm{d} Z^{*}(s)=-\theta Z^{*}(s) \mathrm{d} \tilde{W}_{1}(s)=-\theta Z^{*}(s) \frac{1}{\sigma_{1} S(s)} \mathrm{d} S(s)=-\frac{\theta}{\sigma_{1} S(s)} \mathrm{d} S(s)=: \frac{\zeta(s)}{Z^{*}(s)} \mathrm{d} S(s)
$$

where $\zeta(s)=-\theta Z^{*}(s) / \sigma_{1} S(s)=-\eta(s) Z^{*}(s)$.

Remark 3.1. The variance optimal martingale measure $P^{*}$ in our model coincides with the minimal martingale measure $Q . \tilde{W}$ given in the last of Section 2.2, is also two-dimensional Brownian motion under $P^{*}$.

\subsection{Mean-Variance Hedging for a Unit of Claim}

In this section we give the mean-variance hedging strategy. To this end, we first derive the perfect hedging strategy for the claim $H$ under VOMM $P^{*}$ by reference to [17].

The value processes $S$ and $Y$ are respectively driven by

$$
\begin{gathered}
\mathrm{d} \hat{S}(t)=\sigma_{1} \hat{S}(t) \mathrm{d} W_{1}(t) \\
\mathrm{d} Y(t)=\left(\mu_{2}-\rho \theta \sigma_{2}\right) Y(t) \mathrm{d} t+\sigma_{2} Y(t) \mathrm{d} \tilde{W}_{2}(t)
\end{gathered}
$$

under $P^{*}$, where $\mathrm{d} \tilde{W}_{2}=\rho \mathrm{d} \tilde{W}_{1}+\sqrt{1-\rho^{2}} \mathrm{~d} \tilde{W}^{\perp}$. The Galtchouk-Kunita-Watanabe decomposition for $H \in \mathcal{L}^{2}(P)$ under $P^{*}$ is then given by

$$
H(T)=E^{*}\left[H(T) \mid \mathcal{F}_{0}\right]+\int_{0}^{T} \xi^{H, P^{*}} \mathrm{~d} \hat{S}(s)+L^{H, P^{*}}(T)=: V^{H, P^{*}}(T)
$$

with

$$
V^{H, P^{*}}(t)=E^{*}\left[H(T) \mid \mathcal{F}_{0}\right]+\int_{0}^{t} \xi^{H, P^{*}} \mathrm{~d} \hat{S}(s)+L^{H, P^{*}}(t), 0 \leq t \leq T .
$$

Both of $\int_{0}^{\cdot} \xi^{H, P^{*}} \mathrm{~d} \hat{S}(s)$ and $L^{H, P^{*}}$ are martingales under $P^{*}, \int_{0} \xi^{H, P^{*}} \mathrm{~d} \hat{S}(s)$ is orthogonal to $L^{H, P^{*}}$ under $P^{*}$.

Remark 3.2. In the case of $k$ units claim $(k>1)$, the Galtchouk-Kunita-Watanabe decomposition is directly given by

$$
k H(T)=E^{*}\left[k H(T) \mid \mathcal{F}_{0}\right]+\int_{0}^{T} \xi^{k H, P^{*}} \mathrm{~d} \hat{S}(s)+L^{k H, P^{*}}(T)=: V^{k H, P^{*}}(T)
$$


with

$$
V^{k H, P^{*}}(t)=E^{*}\left[k H(T) \mid \mathcal{F}_{0}\right]+\int_{0}^{t} \xi^{k H, P^{*}} \mathrm{~d} \hat{S}(s)+L^{k H, P^{*}}(t), 0 \leq t \leq T .
$$

from (3.5) and (3.6), where $\xi^{k H, P^{*}}=k \xi^{H, P^{*}}$ and $L^{k H, P^{*}}=k L^{H, P^{*}}$.

Now we solve $\xi^{H, P^{*}}$ and $L^{H, P^{*}}$. Put

$$
v^{*}(t, \hat{S}(t), Y(t))=E^{*}\left[H \mid \mathcal{F}_{t}\right]
$$

from Markov property. Feynman-Kac formula yields that $v^{*}(t, \hat{S}(t), Y(t))$ is a solution of

$$
\partial_{t} v^{*}+\left(\mu_{2}-\rho \theta \sigma_{2}\right) y \partial_{y} v^{*}+\frac{1}{2} \sigma_{1}^{2} x^{2} \partial_{S S} v^{*}+\sigma_{1} \sigma_{2} \hat{S} y \partial_{S y} v^{*}+\frac{1}{2} \sigma_{2}^{2} y^{2} \partial_{y y} v^{*}=0
$$

with $v^{*}(T, \hat{S}, y)=H$, where $\partial_{z} f=\partial f(z) / \partial z$. On the other hand, by Ito's formula, we have

$$
\begin{aligned}
\mathrm{d} v^{*}= & \left(\partial_{t} v^{*}+\left(\mu_{2}-\rho \theta \sigma_{2}\right) y \partial_{y} v^{*}+\frac{1}{2} \sigma_{1}^{2} x^{2} \partial_{S S} v^{*}+\sigma_{1} \sigma_{2} \hat{S} y \partial_{S y} v^{*}+\frac{1}{2} \sigma_{2}^{2} y^{2} \partial_{y y} v^{*}\right) \mathrm{d} t \\
& +\partial_{S} v^{*} \mathrm{~d} \hat{S}(t)+\sigma_{2} y \partial_{y} v^{*} \mathrm{~d} \tilde{W}_{2}(t) .
\end{aligned}
$$

Substituting (3.7) into (3.8) we obtain

then it holds

$$
\mathrm{d} v^{*}(t, \hat{S}(t), Y(t))=\partial_{s} v^{*}(t, \hat{S}(t), Y(t)) \mathrm{d} \hat{S}(t)+\sigma_{2} y \partial_{y} v^{*}(t, \hat{S}(t), Y(t)) \mathrm{d} \tilde{W}_{2}(t),
$$

$$
v^{*}(t, \hat{S}(t), Y(t))=v^{*}(0, \hat{S}, y)+\int_{0}^{t} \partial_{s} v^{*}(s, \hat{S}(s), Y(s)) \mathrm{d} \hat{S}(s)+\int_{0}^{t} \sigma_{2} Y(s) \partial_{y} v^{*}(s, \hat{S}(s), Y(s)) \mathrm{d} \tilde{W}_{2}(s),
$$

By comparison between (3.6) and (3.10), we have $\xi^{H, P^{*}}$ and $L^{H, P^{*}}$ as

$$
\begin{gathered}
\xi^{H, P^{*}}(t)=\partial_{S} v^{*}(t, \hat{S}(t), Y(t))+\frac{\rho \sigma_{2} Y(t)}{\sigma_{1} \hat{S}(t)} \partial_{y} v^{*}(t, \hat{S}(t), Y(t)), \\
L^{H, P^{*}}(t)=\int_{0}^{t} \sigma_{2} \sqrt{1-\rho^{2}} Y(s) \partial_{y} v^{*}(s, \hat{S}(s), Y(s)) \mathrm{d} W^{\perp}(s) .
\end{gathered}
$$

Theorem 3.1. (Schweizer [17]) The mean-variance hedging strategy $\left(C, \kappa^{*}\right)$ for $H \in \mathcal{L}_{2}(P)$ is given by

$$
\begin{gathered}
C=V^{H, P^{*}}(0)=E^{*}[H(T)] \\
\kappa^{*}(t)=\xi^{H, P^{*}}(t)+\eta(t)\left(V^{H, P^{*}}(t)-C-G^{m v h}(t)\right)
\end{gathered}
$$

for $0 \leq t \leq T$, where $\eta(t)=\theta / \sigma_{1} S(t)$ and $G^{m v h}$ is the gain process for the mean-variance hedging strategy $\kappa^{*}$, i.e., $G^{m v h}(t)=\int_{0}^{t} \kappa^{*}(s) \mathrm{d} \hat{S}(s)$.

\subsection{Mean-Variance Hedging for Multi-Volume Claims}

In this section, we construct the mean-variance hedging strategy for the claim extending units of claim to multiple volumes.

Proposition 3.2. The mean-variance hedging strategy for k-claims is given by

$$
\begin{gathered}
C^{(k)}=V^{k H, P^{*}}(0)=k E^{*}[H(T)] \\
\kappa^{(k)^{*}}(t)=\xi^{k H, P^{*}}(t)+\eta(t)\left(V^{k H, P^{*}}(t)-C^{(k)}-G^{m v h(k)}(t)\right)
\end{gathered}
$$

for $0 \leq t \leq T$.

Proof. For a constant $k, k H$ remains in $\mathcal{L}_{2}(P)$. Therefore, from the first assertion of Theorem 3.1, the initial cost of the mean-variance hedging strategy is given by 


$$
C^{(k)}=E^{*}[k H]=k E^{*}[H] .
$$

Next, we verify that $\kappa^{(k)^{*}}$ is the mean-variance hedging strategy. To do this, we set

$$
I(t)=E\left[\left(V^{k H, P^{*}}(t)-C^{(k)}-G^{m v h(k)}(t)\right) G^{(k)}(t)\right]
$$

for $0 \leq t \leq T$. From Lemma 1 in [13], the optimality of $\kappa^{(k)^{*}}$ is equivalent to satisfy

$$
I(t)=0 \text {. }
$$

Defining $D(t):=V^{k H, P^{*}}(t)-C^{(k)}-G^{m v h(k)}(t)$ with $D(0)=0$, leads

$$
\begin{aligned}
\mathrm{d} D(t) & =\mathrm{d} V^{k H, P^{*}}(t)-\mathrm{d} G^{m v h(k)}(t) \\
& =\xi^{H, P^{*}}(t) \mathrm{d} \hat{S}(t)+\mathrm{d} L^{k H, P^{*}}(t)-\kappa^{(k)^{*}}(t) \mathrm{d} X(t) \\
& =\left(\xi^{H, P^{*}}(t)-\kappa^{(k)^{*}}\right) \mathrm{d} \hat{S}(t)+\mathrm{d} L^{k H, P^{*}}(t) \\
& =-\eta(t) D(t) \mathrm{d}(M(t)+A(t))+\mathrm{d} L^{k H, P^{*}}(t) .
\end{aligned}
$$

From Ito's formula and the orthogonal relation between $\int \kappa^{(k)^{*}} \mathrm{~d} \hat{S}(t)$ and $L^{k H, P^{*}}$, we have

$$
\begin{aligned}
\mathrm{d}\left(D(t) G^{(k)}(t)\right) & =D(t) \mathrm{d} G^{(k)}(t)+V^{(k)}(t) \mathrm{d} D(t)+\mathrm{d}\left\langle D, G^{(k)}\right\rangle(t) \\
& =D(t) \kappa^{(k)}(t) \mathrm{d} A(t)-\eta(t) D(t) G^{(k)}(t) \mathrm{d} A(t)-D(t) \kappa^{(k)}(t) \mathrm{d} A(t)+\text { (martingales) } \\
& =-\eta(t) D(t) G^{(k)}(t) \mathrm{d} A(t)+(\text { martingales }) \\
& =-\theta^{2} D(t) G^{(k)}(t) \mathrm{d} t+\text { (martingales). }
\end{aligned}
$$

From Fubini's theorem, we obtain

$$
\begin{aligned}
I(t) & =E\left[D(t) G^{(k)}(t)\right]=E\left[\int_{0}^{t} \theta^{2} D(s) G^{(k)}(s) \mathrm{d} s\right] \\
& =\int_{0}^{t}\left(-\theta^{2}\right) E\left[D(s) G^{(k)}(s)\right] \mathrm{d} s=-\int_{0}^{t} \theta^{2} I(s) \mathrm{d} s
\end{aligned}
$$

since $\theta$ is deterministic. So it holds that

$$
\mathrm{d} I(t)=-\theta^{2} I(t) \mathrm{d} t, 0 \leq t \leq T .
$$

This yields

$$
I(t)=0,0 \leq t \leq T
$$

with $I(0)=0$, and shows (3.13).

\subsection{Hedge Error Risk}

In this section, we solve the hedging error risk measured by $R^{(k)}$ in (2.1). At first, we introduce the hedge error risk amount $R^{(1)}$ for a unit of claim from a previous research.

Theorem 3.2. (Heath et al., [17]) The hedging error amount $R^{(1)}$ defined by (2.1) for a unit of claim $H \in \mathcal{L}^{2}(P)$ is given by

$$
R^{(1)}=\left(E\left[Z^{*}(T) \int_{0}^{T} \frac{1}{Z^{*}(s)} \mathrm{d} L^{H, P^{*}}(s)\right]^{2}\right)^{\frac{1}{2}} .
$$

Let us consider the hedge error risk for multiple units of claim $H$. Define

$$
D(t):=V^{k H, P^{*}}(t)-C^{(k)}-G^{m v h(k)}(t)
$$


again. Then $R^{(k)}=\left(E[D(T)]^{2}\right)^{1 / 2}$ and

$$
\mathrm{d} D(t)=\frac{\theta}{\sigma_{1} \hat{S}(t)} D(t) \mathrm{d} \hat{S}(t)+\mathrm{d} L^{k H, P^{*}}(t)=\frac{\zeta^{*}(t)}{Z^{*}(t)} D(t) \mathrm{d} \hat{S}(t)+\mathrm{d} L^{k H, P^{*}}(t)
$$

with $D(0)=0$, where $\zeta^{*}(s)=-\theta Z^{*}(s) / \sigma_{1} \hat{S}(s)$. The solution of (3.14) is represented by

$$
D(t)=Z^{*}(T) \int_{0}^{t} \frac{1}{Z^{*}(s)} \mathrm{d} L^{k H, P^{*}}(s)
$$

for $0 \leq t \leq T$. This can be checked by applying Ito's product rule to (3.15) from orthogonality of $Z^{*}$ and $\int\left(1 / Z^{*}\right) \mathrm{d} L$.

From (3.15), Remark 3.2 and Theorem 3.2, it holds that

$$
R^{(k)}=\left(E\left[Z^{*}(T) \int_{0}^{T} \frac{1}{Z^{*}(s)} \mathrm{d} L^{k H, P^{*}}(s)\right]^{2}\right)^{\frac{1}{2}}=k\left(E\left[Z^{*}(T) \int_{0}^{T} \frac{1}{Z^{*}(s)} \mathrm{d} L^{H, P^{*}}(s)\right]^{2}\right)^{\frac{1}{2}}=k R^{(k)} .
$$

Therefore, we obtain the following result from (3.16).

Theorem 3.3. The risk amount measured by $R^{(k)}$ for the mean-variance hedging strategy varies in a linear way with respect to the claim volume $k$, i.e.,

$$
\frac{R^{(k)}}{R^{(1)}}=k
$$

\section{Exponential Hedging and Utility Indifference Price}

In this section, we construct the exponential hedging strategy based on the utility indifference price for multiple units of claim. The former has already demonstrated by [5] [6] for a unit of claim, the indifference price is used as the initial hedging cost.

\subsection{Utility Indifference Price with Exponential Utility}

In this section, we derive the utility indifference price as the initial hedging cost in the exponential hedging. The indifference price is derived by solving two distinct utility maximization problems. The one is so-called Merton's problem to maximize the expected utility from the terminal portfolio value, the other is one from terminal portfolio value equipped with claims. Delbaen et al. [1] and Monoyios [5] [6] considered the latter problem as the exponential hedging, in particular [5] [6] derived a hedging strategy for the claim.

In order to derive the utility indifference price, we set utility maximization problems. The market participant has an exponential utility with the risk averse coefficient $\gamma>0$ as follows:

$$
U(x)=-\frac{1}{\gamma} \mathrm{e}^{-\gamma x}
$$

for $x>0$. Set the portfolio strategy $\pi:=\kappa S$, then $\pi$ means the money amount held in the stock. We use $\pi$ as an optimizer of the following utility maximization problems in this section for convenience. The portfolio value process is thus given by

$$
\mathrm{d} X(t)=\frac{\pi(t)}{S(t)} \mathrm{d} S(t) .
$$

We denote the set of all admissible policies $\pi$ for all $\kappa \in \mathcal{A}$ by $\mathcal{A}^{\prime}$.

The problem to maximize the expected utility from terminal portfolio value is given by

$$
u_{0}(t, x)=\sup _{\pi \in \mathcal{A}^{\prime}} E_{t}[U(X(T))]
$$

where $E_{t}$ denotes the expectation conditioned with the market information $\mathcal{F}_{t}$ up to time $t$. On the other hand, 
the problem to maximize the expected utility from terminal portfolio value with $k$ claims is represented by

$$
u(t, x, y)=\sup _{\pi \in \mathcal{A}^{\prime}} E_{t}[U(X(T)-k H(T))] .
$$

This is the value function for the exponential hedging introduced in [1]. We then define the utility indifference price $p(t ; k)$ for $k$ claims.

Definition 4.1. (Utility Indifference Price) The utility indifference price $p(t ; k)$ for $k$ claims at time $t$ is a solution of

$$
u_{0}(t, x)=u(t, x+p(t ; k), y) .
$$

Since the investor receives the premium $p$ at the initial time, so $p$ in Definition 4.1 implies the seller's price. As argued in Section 5.3.2 in [4], it holds that

$$
\lim _{k \rightarrow+0} \frac{p(0 ; k)}{k}=\lim _{\gamma \rightarrow+0} p(0 ; 1)=\tilde{E}[H(T)]
$$

with the exponential utility for the general incomplete market, where $\tilde{E}$ denotes the expectation under $Q$. Theorem 3.1 with (4.2) immediately yields the following theorem.

Theorem 4.1. The utility indifference price coincides with the mean-variance cost for small risk-aversion and claim volume in our basis-risk model.

The basis-risk model permits the explicit solutions for $u_{0}$ and $u$ respectively with the exponential utility, this leads explicit representation of $p$ such as [7].

Proposition 4.1. The value function $u$ is given by

$$
u_{0}(t, x)=-\frac{1}{\gamma} \mathrm{e}^{-\gamma x-\frac{1}{2} \theta^{2}(T-t)}
$$

where $\theta:=\mu_{1} / \sigma_{1}$.

Proof. Hamilton-Jacobi-Bellman (HJB) equation of the value function $u_{0}$ is

$$
\left\{\begin{array}{l}
\partial_{t} u_{0}+\max _{\pi}\left[\pi \mu_{1} \partial_{x} u_{0}+\frac{1}{2} \pi^{2} \sigma_{1}^{2} \partial_{x x} u_{0}\right]=0, \\
u_{0}(T, x)=-\frac{1}{\gamma} \mathrm{e}^{-\gamma x} .
\end{array}\right.
$$

The first order condition leads that the maximum of (4.3) is achieved at

$$
\pi^{*}=-\frac{\theta}{\sigma_{1}} \frac{\partial_{x} u_{0}}{\partial_{x x} u_{0}} .
$$

Substituting $\pi^{*}$ into (4.3) yields the following PDE.

$$
\left\{\begin{array}{l}
\partial_{t} u_{0}-\frac{1}{2} \theta^{2} \frac{\left(\partial_{x} u_{0}\right)^{2}}{\partial_{x x} u_{0}}=0, \\
u_{0}(T, x)=-\frac{1}{\gamma} \mathrm{e}^{-\gamma x} .
\end{array}\right.
$$

Now we set

$$
u_{0}(t, x)=-\frac{1}{\gamma} \mathrm{e}^{-\gamma x} f_{0}(t)
$$

where $f_{0}:[0, T] \mapsto \mathbb{R}$, and plugging this into (4.4) gives

$$
f_{0}^{\prime}(t)=-\frac{1}{2} \theta^{2}(T-t)
$$

This yields 


$$
f_{0}(t)=\exp \left(-\frac{1}{2} \theta^{2}(T-t)\right)
$$

with the terminal condition $f_{0}(T)=1$. From (4.5), the proof is completed.

Q.E.D.

On the other hand, the explicit solution of $u(t, x, y)$ is solved by using an approach demonstrated by some literatures such as [5] [7].

Proposition 4.2. $u(t, x, y)$ is represented by

$$
u(t, x, y)=-\frac{1}{\gamma} \mathrm{e}^{-\gamma x-\frac{1}{2} \theta^{2}(T-t)}\left(\tilde{E}_{t}\left[e^{\left(1-\rho^{2}\right) \gamma k H(T)}\right]\right)^{\frac{1}{1-\rho^{2}}},
$$

where $\tilde{E}$ denotes the expectation under the measure $Q$.

Proof. HJB equation of the value function $u$ is

$$
\left\{\begin{array}{l}
\partial_{t} u+\frac{1}{2} y^{2} \sigma_{2}^{2} \partial_{y y} u+\max _{\pi}\left[\pi \mu_{1} \partial_{x} u+\pi \rho \sigma_{1} \sigma_{2} y \partial_{x y} u+\frac{1}{2} \pi^{2} \sigma_{1}^{2} \partial_{x x} u\right]=0, \\
u(T, x, y)=-\frac{1}{\gamma} \mathrm{e}^{-\gamma(x-k H)} .
\end{array}\right.
$$

The maximum of (4.6) is attained by

$$
\pi^{*}=-\frac{\theta}{\sigma_{1}} \frac{\partial_{x} u}{\partial_{x x} u}-\frac{\rho \sigma_{2} y}{\sigma_{1}} \frac{\partial_{x y} u}{\partial_{x x} u} .
$$

Substituting $\pi^{*}$ into (4.6), we have the following PDE.

$$
\left\{\begin{array}{l}
\partial_{t} u+\frac{1}{2} y^{2} \sigma_{2}^{2} \partial_{y y} u-\frac{1}{2} \theta^{2} \frac{\left(\partial_{x} u\right)^{2}}{\partial_{x x} u}-\rho \theta \sigma_{2} y \frac{\partial_{x} u \partial_{x y} u}{\partial_{x x} u}-\frac{1}{2} \rho^{2} \sigma_{2}^{2} y^{2} \frac{\left(\partial_{x y} u\right)^{2}}{\partial_{x x} u}=0, \\
u(T, x, y)=-\frac{1}{\gamma} \mathrm{e}^{-\gamma(x-k H)} .
\end{array}\right.
$$

By [13], $u$ is represented by

$$
u(t, x, y)=-\frac{1}{\gamma} \mathrm{e}^{-\gamma x} f(t, y)^{\frac{1}{1-\rho^{2}}},
$$

where $f:[0, T] \times \mathbb{R}_{++} \mapsto \mathbb{R}$ solves to

$$
\left\{\begin{array}{l}
-\partial_{t} f+\frac{1}{2}\left(1-\rho^{2}\right) \theta^{2} f=-\rho \theta \sigma_{2} y \partial_{y} f+\frac{1}{2} y^{2} \sigma_{2}^{2} \partial_{y y} f, \\
f(T, y)=\mathrm{e}^{\left(1-\rho^{2}\right) \gamma k H} .
\end{array}\right.
$$

Feynman-Kac formula yields that

$$
f(t, y)=\mathrm{e}^{-\frac{1}{2}\left(1-\rho^{2}\right) \theta^{2}(T-t)} \tilde{E}_{t}\left[\mathrm{e}^{\left(1-\rho^{2}\right) \gamma k H}\right] .
$$

Plugging this into (4.9) concludes the proof.

Proposition 4.3. The utility indifference price $p(t ; k)$ at time $t$ for $k$ units of claim is

$$
p(t ; k)=\frac{1}{\gamma\left(1-\rho^{2}\right)} \ln \tilde{E}_{t}\left[\mathrm{e}^{\left(1-\rho^{2}\right) \gamma k H}\right] .
$$

\subsection{Exponential Hedging}

The exponential hedging has been considered by Delbaen et al. [1], the value function of the hedging problem 
arises in the utility indifference price approach with the exponential utility, i.e., $u(t, x, y)$. Our study extends Delbaen et al.'s problem to the problem with multiple units of claim. Furthermore, Monoyios [5] [6] derived the hedging strategy for the claim as Delta hedge. The hedging strategies demonstrated by [5] [6] take into account the initial hedging cost which is the utility indifference price. We thus apply Monoyios's works [5] [6] to our hedging problem.

Proposition 4.4. The exponential hedging strategy $\delta$ held in the stock is given by

$$
\delta(t)=\frac{\rho \sigma_{2} y}{\sigma_{1} S} \partial_{y} p(t ; k) .
$$

Proof. See [10].

Q.E.D.

Then, we define the hedge error for the exponential hedging as introduced in Section 3. The risk amount of the hedge error $R^{(k)}$ for $k$-claims with the exponential hedging is represented by

$$
R^{(k)}=\left(k H(T)-p(0 ; k)-\int_{0}^{T} \delta(s) \mathrm{d} S(s)\right)^{\frac{1}{2}} .
$$

\subsubsection{Asymptotic Expansion of Exponential Hedging Strategy}

Let us derive an asymptotic expansion of the exponential hedging strategy, i.e., $\delta$ in (4.11). Since we have no closed formula for $p(t ; k)$ in (4.10), it is convenient to use the asymptotic formula of $p(t ; k)$ to obtain a closed formula of the hedging strategy. Monoyios [5] has respectively derived an asymptotic expansion of $p(t ; k)$ and $\delta$ when $k=1$ by the power series expansion scheme, we also provide those for the case of $k>1$ by same manner.

Proposition 4.5. The utility indifference price $p(t ; k)$ for $k$-claims $(k>1)$ with an exponential utility is represented by

$$
p(t ; k)=k\left\{\tilde{E}_{t}[H(T)]+\frac{1}{2} \varepsilon \widetilde{\operatorname{Var}}_{t}[H(T)]+O\left(\varepsilon^{2}\right)\right\},
$$

where $\varepsilon:=\gamma\left(1-\rho^{2}\right) k$ and $\widetilde{\operatorname{Var}}_{t}$ denotes the variance operator under Q-measure conditioned with the information up to $t$, if the parameters satisfy

$$
\tilde{E}\left[\mathrm{e}^{\varepsilon H(T)}\right] \leq 2 .
$$

Proof. Taylor expansion for $f(x)=\mathrm{e}^{a x}$ ( $a$ is a constant) is

$$
f(x)=1+a x+\frac{1}{2 !} a^{2} x^{2}+O\left(a^{3}\right)
$$

where $O(a)$ denotes terms proportional to $a$. Therefore, (4.10) is rewritten by

$$
p(t ; k)=\frac{k}{\varepsilon} \ln \tilde{E}_{t}\left[\mathrm{e}^{\varepsilon H(T)}\right]=\frac{k}{\varepsilon} \ln \left\{1+\varepsilon \tilde{E}_{t}[H(T)]+\frac{1}{2 !} \varepsilon^{2} \tilde{E}_{t}\left[H^{2}(T)\right]+O\left(\varepsilon^{3}\right)\right\} .
$$

The power series expansion for $g(x)=\ln (1+x)$ is given by

$$
\ln (1+x)=x-\frac{1}{2} x^{2}+O\left(x^{3}\right)
$$

with $-1 \leq x \leq 1$ (or equivalently $0 \leq 1+x \leq 2$ ). Applying (4.14) to (4.15) for the condition of (4.13), we have

if $\tilde{E}\left[\mathrm{e}^{\varepsilon H(T)}\right] \leq 2$.

$$
p(t ; k)=\frac{k}{\varepsilon}\left\{\varepsilon \tilde{E}_{t}[H(T)]+\frac{1}{2 !} \varepsilon^{2}\left(\tilde{E}_{t}\left[H^{2}(T)\right]-\tilde{E}_{t}[H(T)]^{2}\right)+O\left(\varepsilon^{3}\right)\right\},
$$

From Proposition 4.5, we obtain a closed formula of the exponential hedging strategy (4.11) by calculating the first derivative of (4.12). From (4.12), we have 


$$
\partial_{y} p(t ; k)=k\left\{\partial_{y} \tilde{E}_{t}[H(T)]+\frac{1}{2 !} \varepsilon\left(\partial_{y} \tilde{E}_{t}\left[H^{2}(T)\right]-2 \tilde{E}_{t}[H(T)] \partial_{y} \tilde{E}_{t}[H(T)]\right)+O\left(\varepsilon^{3}\right)\right\} .
$$

\section{Numerical Example and Main Result}

In this section, we demonstrate the exponential hedging discussed above by using Monte-Carlo simulation.

We also obtain main results of this work through the numerical simulations in this section.

\section{Main Result}

As mentioned in Section 2, we consider the hedging problems for the put option written on $Y$. Its payoff function is

with the strike price $K$.

$$
H(T):=H(T, Y(T))=(K-Y(T))^{+}
$$

For the claim $H$ presented by (5.1), we have

$$
\begin{aligned}
\tilde{E}_{t}[H(T)]= & K \Phi\left(-d_{2}(t)\right)-Y(t) \mathrm{e}^{\left(\mu_{2}-\rho \theta \sigma_{2}\right)(T-t)} \Phi\left(-d_{1}(t)\right), \\
\tilde{E}_{t}\left[H^{2}(T)\right]= & K^{2} \Phi\left(-d_{2}(t)\right)-2 K Y(t) \mathrm{e}^{\left(\mu_{2}-\rho \theta \sigma_{2}\right)(T-t)} \Phi\left(-d_{1}(t)\right) \\
& +Y^{2}(t) \mathrm{e}^{2\left(\mu_{2}-\rho \theta \sigma_{2}+\frac{1}{2} \sigma_{2}^{2}\right)(T-t)} \Phi\left(-d_{1}(t)-\sigma_{2} \sqrt{T-t}\right)
\end{aligned}
$$

where $\Phi$ denotes the distribution function of the standard normal distribution and

And also, from the fact that

$$
\begin{gathered}
d_{1}(t)=\frac{\ln \left(\frac{Y(t)}{K}\right)+\left(\mu_{2}-\rho \theta \sigma_{2}+\frac{1}{2} \sigma_{2}^{2}\right)(T-t)}{\sigma_{2} \sqrt{T-t}}, \\
d_{2}(t)=d_{1}(t)-\sigma_{2} \sqrt{T-t} .
\end{gathered}
$$

$$
Y(t) \mathrm{e}^{\left(\mu_{2}-\rho \theta \sigma_{2}\right)(T-t)} \Phi^{\prime}\left(-d_{1}(t)\right)=K \Phi^{\prime}\left(-d_{2}(t)\right),
$$

it holds

$$
\begin{gathered}
\partial_{y} \tilde{E}_{t}[H(T)]=-\mathrm{e}^{\left(\mu_{2}-\rho \theta \sigma_{2}\right)(T-t)} \Phi\left(-d_{1}(t)\right), \\
\partial_{y} \tilde{E}_{t}\left[H^{2}(T)\right]=2\left\{Y(t) \mathrm{e}^{2\left(\mu_{2}-\rho \theta \sigma_{2}+\frac{1}{2} \sigma_{2}^{2}\right)(T-t)} \Phi\left(-d_{1}(t)-\sigma_{2} \sqrt{T-t}\right)-K \mathrm{e}^{\left(\mu_{2}-\rho \theta \sigma_{2}\right)(T-t)} \Phi\left(-d_{1}(t)\right)\right\}
\end{gathered}
$$

We obtain the exponential hedging strategy in the closed form by substituting these into (4.16).

We first should check whether our model and parameters satisfy the condition (4.13). We specially select the upper of $k$ to satisfy the condition (4.13). We use parameters described in Table 1. Table 2 summarizes the simulated results for $\tilde{E}_{t}\left[\mathrm{e}^{\varepsilon H(T)}\right]$, its standard error when $\gamma=0.01$ and its upper bound. The upper bound of $\tilde{E}_{t}\left[\mathrm{e}^{\varepsilon H(T)}\right]$ is defined as the upper value of $95 \%$ confidence interval for $\tilde{E}_{t}\left[\mathrm{e}^{\varepsilon H(T)}\right]$, i.e.,

$$
\text { Upper bound of } \tilde{E}_{t}\left[\mathrm{e}^{\varepsilon H(T)}\right]:=\text { Mean }+1.96 \times \frac{\text { Std.Error }}{\sqrt{N}}
$$

where $N$ is the number of simulation times. And then, we simulate $\tilde{E}_{t}\left[\mathrm{e}^{\varepsilon H(T)}\right]$ up to $k$ such that

$$
\text { Upper bound of } \tilde{E}_{t}\left[e^{\varepsilon H(T)}\right] \leq 2 \text {. }
$$

The table shows that our parameters are valid up to $k=10$. The fact that $\tilde{E}_{t}\left[\mathrm{e}^{\varepsilon H(T)}\right]$ is monotone increasing with respect to $\gamma$, allows us to use the above asymptotic rule for $\gamma \leq 0.01$. 
Tables 3(a)-(d) show the risk amount $R^{(k)}$ of the hedge error and its simulation error (denoted by "Bound") for the mean-variance hedging Table 3(a) and the exponential hedging Tables 3(b)-(d). As to the exponential hedging, we simulate the hedging strategy for $\gamma=0.001,0.005,0.01$. The bound of $R^{(k)}$ is calculated as a $95 \%$ confidence interval such that

$$
\left[\text { Mean }-1.96 \times \frac{\text { Std.Error }}{\sqrt{N}} \text {, Mean }+1.96 \times \frac{\text { Std.Error }}{\sqrt{N}}\right] .
$$

Needless to say, the hedge error risk increases according to the claim volume $k$ for both hedging strategies. We are however interested in the increment of the risk amount rather than itself. That is, how the risk amount increases according to the claim volume $k$ ? To this end, we evaluate the proportion of the risk amount for multivolume traded to the risk amount for a unit claim sold, i.e., $R^{(k)} / R^{(1)}$. Table 4 shows the proportion of the risk amount $R^{(k)} / R^{(1)}$ for the exponential hedging and results are described in Figure 1. At first, the risk amount increases in linear way for all $k$ at $\gamma=0.001$, and so do up to $k=6$ at $\gamma=0.005$, respectively. On the other hand, at $\gamma=0.01$, it linearly increases up to $k=3$, and it increases in nonlinear way after that. We note that these differences are significant by observing the bound of $R^{(k)}$ in Table 3. This implies that the risk amount tends to increase nonlinearly for large risk averse coefficient. Also, the risk amount linearly increases for small $k$ independent with the level of $\gamma$.

As shown in Theorem 3.3, the risk amount of the hedge error varies in linear way for the mean-variance

Table 1. Parameters used in the numerical examples.

\begin{tabular}{cccccccccc}
\hline Parameter & $S(0)$ & $\mu_{1}$ & $\sigma_{1}$ & $Y(0)$ & $\mu_{2}$ & $\sigma_{2}$ & $K$ & $T$ \\
\hline Value & 100 & 0.01 & 0.25 & 100 & 0.12 & 0.30 & 100 & 1.0 \\
\hline
\end{tabular}

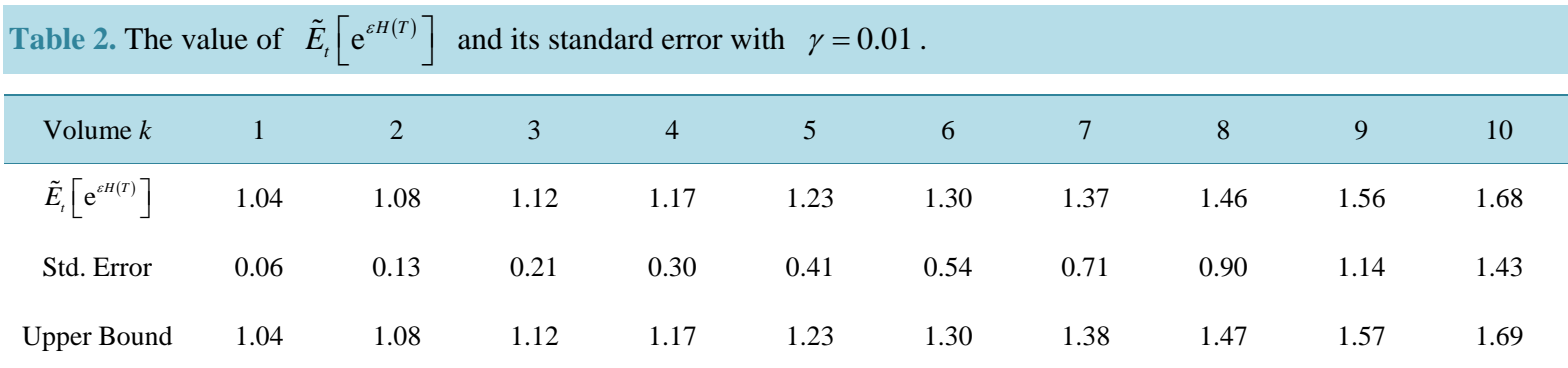

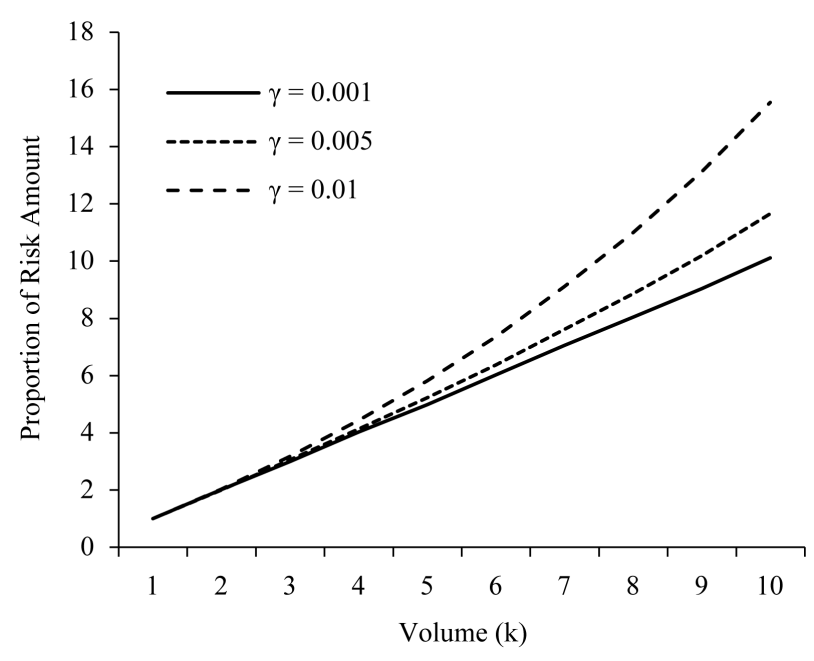

Figure 1. Proportion of the risk amount, $R^{(k)} / R^{(1)}$, for the exponential hedging. 
Table 3. (a) The risk amount of the hedge error $R^{(k)}$ and its bound for the mean-variance hedging; (b) The risk amount of the hedge error $R^{(k)}$ and its bound for the exponential hedging at $\gamma=0.001$; (c) The risk amount of the hedge error $R^{(k)}$ and its bound for the exponential hedging at $\gamma=0.005$; (d) The risk amount of the hedge error $R^{(k)}$ and its bound for the exponential hedging at $\gamma=0.01$.

(a)

\begin{tabular}{ccccccccccc}
\hline Volume $k$ & 1 & 2 & 3 & 4 & 5 & 6 & 7 & 8 & 9 & 10 \\
\hline \multirow{2}{*}{ Risk Amount } & 7.99 & 15.88 & 24.12 & 32.01 & 39.97 & 47.7 & 56.04 & 63.77 & 71.52 & 79.54 \\
& & & & & & & & & & \\
Bound & $7.94-$ & $15.79-$ & $23.98-$ & $31.81-$ & $39.72-$ & $47.41-$ & $55.70-$ & $63.38-$ & $71.09-$ & $79.05-$ \\
& 8.04 & 15.98 & 24.27 & 32.21 & 40.21 & 48.00 & 56.38 & 64.16 & 71.96 & 80.03 \\
\hline
\end{tabular}

a. The second line shows the risk amount of the hedge error $R^{(k)}$, and the third line lists the its confidence interval.

(b)

\begin{tabular}{ccccccccccc}
\hline Volume $k$ & 1 & 2 & 3 & 4 & 5 & 6 & 7 & 8 & 9 & 10 \\
\hline \multirow{2}{*}{ Risk Amount } & 7.99 & 15.9 & 24.15 & 32.09 & 40.07 & 47.91 & 56.31 & 64.14 & 72.02 & 80.27 \\
& & & & & & & & & & \\
Bound & $7.94-$ & $15.80-$ & $24.00-$ & $31.90-$ & $39.83-$ & $47.62-$ & $55.98-$ & $63.76-$ & $71.59-$ & $79.80-$ \\
& 8.04 & 15.99 & 24.29 & 32.29 & 40.31 & 48.20 & 56.64 & 64.52 & 72.44 & 80.74 \\
\hline
\end{tabular}

a. The second line shows the risk amount of the hedge error $R^{(k)}$, and the third line lists the its confidence interval.

(c)

\begin{tabular}{ccccccccccc}
\hline Volume $k$ & 1 & 2 & 3 & 4 & 5 & 6 & 7 & 8 & 9 & 10 \\
\hline \multirow{2}{*}{ Risk Amount } & 8.01 & 16.03 & 24.51 & 33.03 & 41.85 & 50.99 & 61.02 & 70.93 & 81.58 & 93.39 \\
\multirow{2}{*}{ Bound } & $7.96-$ & $15.93-$ & $24.37-$ & $32.85-$ & $41.62-$ & $50.71-$ & $60.69-$ & $70.55-$ & $\begin{array}{c}81.15- \\
82.00\end{array}$ & $92.90-$ \\
& 8.06 & 16.12 & 24.65 & 33.22 & 42.08 & 51.27 & 61.34 & 71.31 & 82.00 \\
\hline
\end{tabular}

a. The second line shows the risk amount of the hedge error $R^{(k)}$, and the third line lists the its confidence interval.

(d)

\begin{tabular}{ccccccccccc}
\hline Volume $k$ & 1 & 2 & 3 & 4 & 5 & 6 & 7 & 8 & 9 & 10 \\
\hline \multirow{2}{*}{ Risk Amount } & 8.05 & 16.38 & 25.61 & 35.67 & 46.8 & 59.27 & 73.46 & 88.6 & 105.73 & 125.22 \\
& & & & & & & & & & \\
Bound & $8.01-$ & $16.29-$ & $25.47-$ & $35.48-$ & $46.55-$ & $58.96-$ & $73.08-$ & $88.15-$ & $105.19-$ & $124.59-$ \\
& 8.10 & 16.47 & 25.75 & 35.87 & 47.05 & 59.58 & 73.83 & 89.05 & 106.26 & 125.85 \\
\hline
\end{tabular}

a. The second line shows the risk amount of the hedge error $R^{(k)}$, and the third line lists the its confidence interval.

Table 4. Proportion of the risk amount, $R^{(k)} / R^{(1)}$, for the exponential hedging.

\begin{tabular}{|c|c|c|c|c|c|c|c|c|c|c|}
\hline Volume $k$ & 1 & 2 & 3 & 4 & 5 & 6 & 7 & 8 & 9 & 10 \\
\hline$\gamma=0.001$ & 1.00 & 2.01 & 3.00 & 4.02 & 4.98 & 6.03 & 7.06 & 8.04 & 9.04 & 10.11 \\
\hline$\gamma=0.005$ & 1.00 & 2.00 & 3.06 & 4.13 & 5.23 & 6.37 & 7.62 & 8.86 & 10.19 & 11.66 \\
\hline$\gamma=0.01$ & 1.00 & 2.03 & 3.18 & 4.43 & 5.81 & 7.36 & 9.12 & 11.00 & 13.13 & 15.55 \\
\hline
\end{tabular}

a. The risk amount tends to increase in nonlinear way according to the claim volume $k$ for large risk-averse coefficient and claim volume.

hedging. Our numerical experiences thus show the convergence of the exponential hedging to the mean-variance hedging about the linear increment of the hedge error risk when the risk-averse coefficient and the claim volumego to zero. In fact, Tables 3(a)-(d) show that the difference in the risk amounts between the mean-variance hedging and the exponential hedging are very small for small risk-aversion $\gamma$ and claim volume $k$. The risk amount of the hedge error for the exponential hedging with $\gamma=0.001$ more closes with the one for the mean-va- 
riance hedging rather than the cases of $\gamma=0.005,0.01$. Such convergence has already been shown by [9]. Also, even in the case of $\gamma=0.01$, the risk amount of the exponential hedging closes to the one of the mean-variance hedging for which $k$ is less than or equal 3.

We further add the characteristics about the increment of the risk amount for the both hedging strategies. In particular, we implement hedging strategies by varying $\rho$. The parameters used in this demonstration are described in Table 5. We use parameters as same as the ones used in the previous section except of $\rho$. At first, we check the validity of the asymptotic formulae for the exponential hedging strategy and the utility indifference price. That is, whether the parameters used described in Table 5 satisfy (5.3) or not, as experienced in the above. Table 6 shows the simulated results for $\tilde{E}_{t}\left[\mathrm{e}^{\varepsilon H(T)}\right]$, its standard error when $\gamma=0.01$ and its upper bound. The upper bound of $\tilde{E}_{t}\left[\mathrm{e}^{\varepsilon H(T)}\right]$ is defined by (5.2). The table indicates that the asymptotic formulae are valid up to $k=5$.

Tables 7(a)-(g) shows the hedge error risk amount for the mean-variance hedging, and the graph is described in Figure 2. From the table or the figure, the hedge error amount is concave with respect to $\rho$ for each claim volume $k$. This property however is violated for the exponential hedging for large claim volume. Tables 8(a)-(g) show the hedge error risk amount for the exponential hedging, and the graph is given in Figure 3. Obviously, the risk amount of the hedge error does not have a concave with respect to $\rho$ at $k \geq 3$. On the other hand, the concavity is preserved for $k \geq 2$.

Furthermore, we consider the linearity of the hedge error risk, the linearity is one of the characteristics of the mean-variance hedging. Tables 9(a)-(g) shows the proportion of the risk amount $R^{(k)} / R^{(1)}$ for each $\rho$, and the graph is given in Figure 4. From the table, the linearity is relatively preserved for around $\rho=0.0$, and it is violated when $\rho$ leaves from zero. For example, at $\rho=-0.75$, the risk proportion increases in a nonlinear way

Table 5. Parameters used in the numerical examples.

\begin{tabular}{ccccccccccc}
\hline Parameter & $S(0)$ & $\mu_{1}$ & $\sigma_{1}$ & $Y(0)$ & $\mu_{2}$ & $\sigma_{2}$ & $K$ & $T$ \\
Value & 100 & 0.01 & 0.25 & 100 & 0.12 & 0.30 & 100 & 1.0 \\
\hline
\end{tabular}

Table 6. The value of $\tilde{E}_{t}\left[\mathrm{e}^{\varepsilon H(T)}\right]$ and its standard error with $\gamma=0.01$ and $\rho=0.0$.

\begin{tabular}{cccccc}
\hline Volume $k$ & 1 & 2 & 3 & 4 & 5 \\
\hline$\tilde{E}_{t}\left[\mathrm{e}^{\varepsilon H(T)}\right]$ & 1.08 & 1.20 & 1.35 & 1.55 & 1.85 \\
Std. Error & 0.14 & 0.35 & 0.67 & 1.14 & 1.91 \\
Upper Bound & 1.08 & 1.20 & 1.35 & 1.55 & 1.85 \\
\hline
\end{tabular}

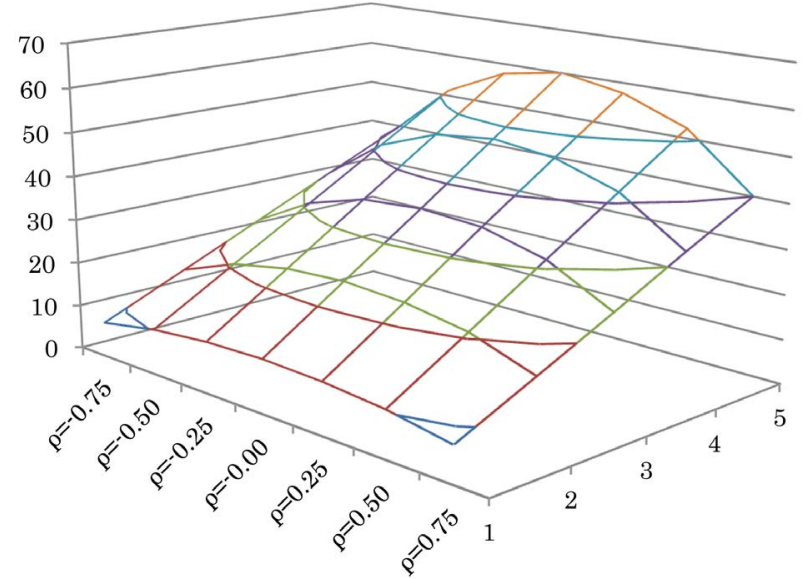

Figure 2. Hedge error risk of the mean-variance hedging for each $\rho$. 


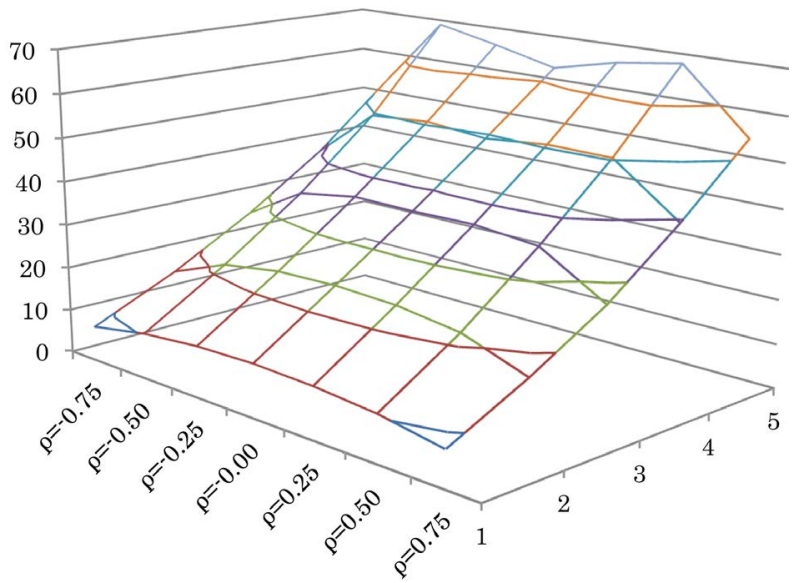

Figure 3. Hedge error risk of the exponential hedging for each $\rho$.

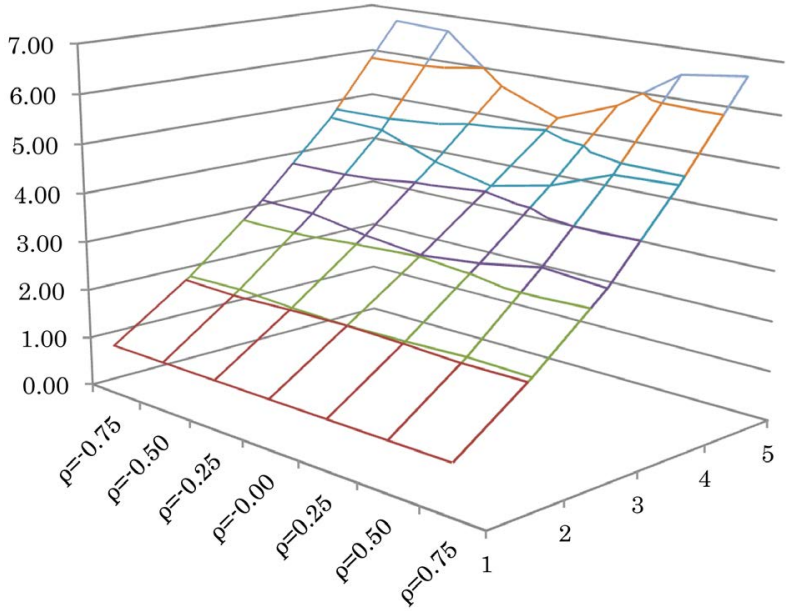

Figure 4. Proportion of the risk amount $R^{(k)} / R^{(1)}$ of the exponential hedging for each $\rho$.

with $k$ for $k \geq 3$. This property also stands in the case of $\rho=0.75$ too. However, the proportion of the hedge error risk amount varies in a linear way for small claim volume even though the case of $\rho<0.0$ or $\rho>0.0$. Therefore, the hedge error risk amount for the exponential hedging tends to have the linearity when the claim volume closes to zero for each $\rho$.

Summarizing results considered in the above, we have the following theorem.

Theorem 5.1. The exponential hedging with the utility indifference price as the initial hedging cost, converges to the mean-variance hedging when the claim volume $k$ or the risk averse coefficient $\gamma$ closes to zero.

\section{Concluding Remarks}

In this work, we constructed both the mean-variance and exponential hedging strategies for multiple units of claim and calculated the hedge error risks for each risk-averse level and claim volume. The hedge error risk is measured by the squared root of the expectation of the quadratic hedge error. We then characterized the nonlinear increment of the hedge error risk with respect to the claim volume for the exponential hedging strategy; that is, the hedge error risk varies in a nonlinear way with respect to the claim volume. By contrast, the hedge error risk changes in the linear way for the mean-variance hedging. Our numerical examinations verified that the nonlinear increment is reduced to the linear increment when the risk-averse coefficient and the claim volume go to zero. That is, we showed that the exponential hedging converges to the mean-variance hedging from the point of 
Table 7. (a) The risk amount of the hedge error $R^{(k)}$ and its bound for the mean-variance hedging at $\rho=-0.75$; (b) The risk amount of the hedge error $R^{(k)}$ and its bound for the mean-variance hedging at $\rho=-0.50$; (c) The risk amount of the hedge error $R^{(k)}$ and its bound for the mean-variance hedging at $\rho=-0.25$; (d) The risk amount of the hedge error $R^{(k)}$ and its bound for the mean-variance hedging at $\rho=0.00$; (e) The risk amount of the hedge error $R^{(k)}$ and its bound for the mean-variance hedging at $\rho=0.25$; (f) The risk amount of the hedge error $R^{(k)}$ and its bound for the mean-variance hedging at $\rho=0.50$; (g) The risk amount of the hedge error $R^{(k)}$ and its bound for the mean-variance hedging at $\rho=0.75$.

(a)

\begin{tabular}{cccccc}
\hline Volume $k$ & 1 & 2 & 3 & 4 & 5 \\
\hline Risk Amount & 7.84 & 15.68 & 23.56 & 31.49 & 39.29 \\
Bound & $7.79-7.89$ & $15.59-15.78$ & $23.42-23.71$ & $31.30-31.69$ & $39.04-39.53$ \\
\hline
\end{tabular}

a. The second line shows the risk amount of the hedge error $R^{(k)}$, and the third line lists the its confidence interval.

(b)

\begin{tabular}{cccccc}
\hline Volume $k$ & 1 & 2 & 3 & 4 & 5 \\
\hline Risk Amount & 10.25 & 20.6 & 30.78 & 41.08 & 51.29 \\
Bound & $10.19-10.31$ & $20.47-20.73$ & $30.59-30.97$ & $40.82-41.33$ & $50.97-51.61$ \\
\hline
\end{tabular}

a. The second line shows the risk amount of the hedge error $R^{(k)}$, and the third line lists the its confidence interval.

(c)

\begin{tabular}{cccccc}
\hline Volume $k$ & 1 & 2 & 3 & 4 & 5 \\
\hline Risk Amount & 11.58 & 23.13 & 34.73 & 46.24 & 57.69 \\
Bound & $11.51-11.66$ & $22.98-23.28$ & $34.51-34.95$ & $45.94-46.53$ & $57.33-58.05$ \\
\hline
\end{tabular}

a. The second line shows the risk amount of the hedge error $R^{(k)}$, and the third line lists the its confidence interval.

(d)

\begin{tabular}{cccccc}
\hline Volume $k$ & 1 & 2 & 3 & 4 & 5 \\
\hline Risk Amount & 12.03 & 23.94 & 35.76 & 47.9 & 60.1 \\
Bound & $11.95-12.10$ & $23.79-24.09$ & $35.53-35.99$ & $47.60-48.21$ & $59.73-60.47$ \\
\hline
\end{tabular}

a. The second line shows the risk amount of the hedge error $R^{(k)}$, and the third line lists the its confidence interval.

(e)

\begin{tabular}{cccccc}
\hline Volume $k$ & 1 & 2 & 3 & 4 & 5 \\
\hline Risk Amount & 11.58 & 23.14 & 34.93 & 46.34 & 57.7 \\
Bound & $11.50-11.65$ & $22.99-23.28$ & $34.71-35.15$ & $46.04-46.63$ & $57.33-58.06$ \\
\hline
\end{tabular}

a. The second line shows the risk amount of the hedge error $R^{(k)}$, and the third line lists the its confidence interval.

(f)

\begin{tabular}{cccccc}
\hline Volume $k$ & 1 & 2 & 3 & 4 & 5 \\
\hline Risk Amount & 10.38 & 20.83 & 31.34 & 41.78 & 52.25 \\
Bound & $10.32-10.45$ & $20.70-20.96$ & $31.14-31.53$ & $41.52-42.04$ & $51.92-52.58$ \\
\hline
\end{tabular}

a. The second line shows the risk amount of the hedge error $R^{(k)}$, and the third line lists the its confidence interval.

(g)

\begin{tabular}{cccccc}
\hline Volume $k$ & 1 & 2 & 3 & 4 & 5 \\
\hline Risk Amount & 8.01 & 15.96 & 23.95 & 32.16 & 40.04 \\
Bound & $7.96-8.06$ & $15.86-16.06$ & $23.80-24.10$ & $31.96-32.36$ & $39.80-40.29$ \\
\hline
\end{tabular}

a. The second line shows the risk amount of the hedge error $R^{(k)}$, and the third line lists the its confidence interval. 
Table 8. (a) The risk amount of the hedge error $R^{(k)}$ and its bound for the exponential hedging at $\rho=-0.75$; (b) The risk amount of the hedge error $R^{(k)}$ and its bound for the exponential hedging at $\rho=-0.50$; (c) The risk amount of the hedge error $R^{(k)}$ and its bound for the exponential hedging at $\rho=-0.25$; (d) The risk amount of the hedge error $R^{(k)}$ and its bound for the exponential hedging at $\rho=0.00$; (e) The risk amount of the hedge error $R^{(k)}$ and its bound for the exponential hedging at $\rho=0.25$; (f) The risk amount of the hedge error $R^{(k)}$ and its bound for the exponential hedging at $\rho=0.50$; (g) The risk amount of the hedge error $R^{(k)}$ and its bound for the exponential hedging at $\rho=0.75$.

(a)

\begin{tabular}{cccccc}
\hline Volume $k$ & 1 & 2 & 3 & 4 & 5 \\
\hline Risk Amount & 7.93 & 16.35 & 26.46 & 38.39 & 53.37 \\
Bound & $7.88-7.98$ & $16.25-16.44$ & $26.08-26.83$ & $38.00-38.77$ & $52.51-54.21$ \\
\hline
\end{tabular}

a. The second line shows the risk amount of the hedge error $R^{(k)}$, and the third line lists the its confidence interval.

(b)

\begin{tabular}{cccccc}
\hline Volume $k$ & 1 & 2 & 3 & 4 & 5 \\
\hline Risk Amount & 10.35 & 21.58 & 34.34 & 49.54 & 68.96 \\
Bound & $10.28-10.41$ & $21.45-21.70$ & $34.09-34.59$ & $49.12-49.96$ & $67.87-70.03$ \\
\hline
\end{tabular}

a. The second line shows the risk amount of the hedge error $R^{(k)}$, and the third line lists the its confidence interval.

(c)

\begin{tabular}{cccccc}
\hline Volume $k$ & 1 & 2 & 3 & 4 & 5 \\
\hline Risk Amount & 11.64 & 23.62 & 36.55 & 50.59 & 65.98 \\
Bound & $11.57-11.71$ & $23.49-23.76$ & $36.35-36.75$ & $50.29-50.88$ & $65.53-66.43$ \\
\hline
\end{tabular}

a. The second line shows the risk amount of the hedge error $R^{(k)}$, and the third line lists the its confidence interval.

(d)

\begin{tabular}{cccccc}
\hline Volume $k$ & 1 & 2 & 3 & 4 & 5 \\
\hline Risk Amount & 12.05 & 24.11 & 36.39 & 49.28 & 62.62 \\
Bound & $11.98-12.12$ & $23.98-24.25$ & $36.20-36.58$ & $49.04-49.52$ & $62.35-62.90$ \\
\hline
\end{tabular}

a. The second line shows the risk amount of the hedge error $R^{(k)}$, and the third line lists the its confidence interval.

(e)

\begin{tabular}{cccccc}
\hline Volume $k$ & 1 & 2 & 3 & 4 & 5 \\
\hline Risk Amount & 11.63 & 23.62 & 36.66 & 50.57 & 65.97 \\
Bound & $11.57-11.70$ & $23.49-23.75$ & $36.45-36.87$ & $50.24-50.90$ & $65.52-66.41$ \\
\hline
\end{tabular}

a. The second line shows the risk amount of the hedge error $R^{(k)}$, and the third line lists the its confidence interval.

(f)

\begin{tabular}{cccccc}
\hline Volume $k$ & 1 & 2 & 3 & 4 & 5 \\
\hline Risk Amount & 10.48 & 21.77 & 35.31 & 50.4 & 67.91 \\
Bound & $10.42-10.54$ & $21.64-21.90$ & $34.53-36.07$ & $49.75-51.03$ & $67.19-68.63$ \\
\hline
\end{tabular}

a. The second line shows the risk amount of the hedge error $R^{(k)}$, and the third line lists the its confidence interval.

(g)

\begin{tabular}{cccccc}
\hline Volume $k$ & 1 & 2 & 3 & 4 & 5 \\
\hline Risk Amount & 8.12 & 16.72 & 26.62 & 39.42 & 53.86 \\
Bound & $8.07-8.17$ & $16.57-16.87$ & $26.45-26.80$ & $38.90-39.94$ & $52.54-55.14$ \\
\hline
\end{tabular}

a. The second line shows the risk amount of the hedge error $R^{(k)}$, and the third line lists the its confidence interval. 
Table 9. (a) Proportion of the risk amount, $R^{(k)} / R^{(1)}$, for the exponential hedging at $\rho=-0.75$; (b) Proportion of the risk amount, $R^{(k)} / R^{(1)}$, for the exponential hedging at $\rho=-0.50$; (c) Proportion of the risk amount, $R^{(k)} / R^{(1)}$, for the exponential hedging at $\rho=-0.25$; (d) Proportion of the risk amount, $R^{(k)} / R^{(1)}$, for the exponential hedging at $\rho=0.00$; (e) Proportion of the risk amount, $R^{(k)} / R^{(1)}$, for the exponential hedging at $\rho=0.25$; (f) Proportion of the risk amount, $R^{(k)} / R^{(1)}$, for the exponential hedging at $\rho=0.50$; (g) Proportion of the risk amount, $R^{(k)} / R^{(1)}$, for the exponential hedging at $\rho=0.75$.

(a)

\begin{tabular}{ccccccc}
\hline Volume $k$ & 1 & 2 & 3 & 4 & 5 \\
\hline$R^{(k)} / R^{(1)}$ & 1.00 & 2.06 & 3.34 & 4.84 & 6.73 \\
\hline
\end{tabular}

(b)

\begin{tabular}{ccccccc}
\hline Volume $k$ & 1 & 2 & 3 & 4 & 5 \\
\hline$R^{(k)} / R^{(1)}$ & 1.00 & 2.09 & 3.32 & 4.79 & 6.67 \\
\hline
\end{tabular}

(c)

\begin{tabular}{cccccc}
\hline Volume $k$ & 1 & 2 & 3 & 4 & 5 \\
\hline$R^{(k)} / R^{(1)}$ & 1.00 & 2.03 & 3.14 & 4.35 & 5.67 \\
\hline
\end{tabular}

(d)

\begin{tabular}{cccccc}
\hline Volume $k$ & 1 & 2 & 3 & 4 & 5 \\
\hline$R^{(k)} / R^{(1)}$ & 1.00 & 2.00 & 3.02 & 4.09 & 5.20 \\
\hline
\end{tabular}

(e)

\begin{tabular}{cccccc}
\hline Volume $k$ & 1 & 2 & 3 & 4 & 5 \\
\hline$R^{(k)} / R^{(1)}$ & 1.00 & 2.03 & 3.15 & 4.35 & 5.67 \\
\hline
\end{tabular}

(f)

\begin{tabular}{cccccc}
\hline Volume $k$ & 1 & 2 & 3 & 4 & 5 \\
\hline$R^{(k)} / R^{(1)}$ & 1.00 & 2.08 & 3.37 & 4.81 & 6.48 \\
\hline
\end{tabular}

(g)

\begin{tabular}{cccccc}
\hline Volume $k$ & 1 & 2 & 3 & 4 & 5 \\
\hline$R^{(k)} / R^{(1)}$ & 1.00 & 2.06 & 3.28 & 4.86 & 6.64 \\
\hline
\end{tabular}

the hedge error view. As mentioned in Section 1, it has been already shown that the utility indifference price with the exponential utility converges to the no arbitrage price when the claim volume goes to zero. Hence our results with the results of the previous researches lead a perspective that the utility indifference price with the exponential utility converges to the mean-variance hedging cost in the basis-risk model.

\section{References}

[1] Davis, M.H.A. (2006) Optimal Hedging with Basis Risk. In: Kabanov, Y., Liptser, R. and Stoyanov, J., Eds., From Stochastic Calculus to Mathematical Finance, Springer-Verlag, Berlin, 169-187. http://dx.doi.org/10.1007/978-3-540-30788-4_8

[2] Delbaen, F., Grandits, P., Rheinlander, T., Samperi, M., Schweizer, M. and Stricker, C. (2002) Exponential Hedging and Entropic Penalties. Mathematical Finance, 12, 99-123. http://dx.doi.org/10.1111/1467-9965.02001

[3] Duffie, D. and Richardson, H.R. (1991) Mean-Variance Hedging in Continuous Time. The Annals of Applied Proba- 
bility, 1, 1-15. http://dx.doi.org/10.1214/aoap/1177005978

[4] Frittelli, M. (2000) The Minimal Entropy Martingale Measure and the Valuation Problem in Incomplete Markets. Mathematical Finance, 10, 39-52. http://dx.doi.org/10.1111/1467-9965.00079

[5] Heath, P., Platen, E. and Schweizer, M. (2001) A Comparison of Two Quadratic Approaches to Hedging in Incomplete Markets. Mathematical Finance, 11, 385-413. http://dx.doi.org/10.1111/1467-9965.00122

[6] Henderson, V. (2002) Valuation of Claims on Nontraded Assets Using Utility Maximization. Mathematical Finance, 12, 351-373. http://dx.doi.org/10.1111/j.1467-9965.2002.tb00129.x

[7] Hodges, S.D. and Neuberger, A. (1989) Optimal Replication of Contingent Claims under Transaction Costs. Review of Futures Markets, 8, 222-239.

[8] Ilhan, A., Jonsson, M. and Sircar, R. (2004) Portfolio Optimization with Derivatives and Indifference Pricing. In: Carmona, R., Ed., Indifference Pricing - Theory and Applications, Princeton University Press, Princeton, 183-210.

[9] Mania, M. and Shweizer, M. (2005) Dynamic Exponential Utility Indifference Valuation. The Annals of Applied Probability, 15, 2113-2143. http://dx.doi.org/10.1214/105051605000000395

[10] Monoyios, M. (2004) Performance of Utility-Based Strategies for Hedging Basis Risk. Quantitative Finance, 4, 245255. http://dx.doi.org/10.1088/1469-7688/4/3/001

[11] Monoyios, M. (2008) Optimal Hedging and Parameter Uncertainty. IMA Journal of Management Mathematics, 18, 331-351. http://dx.doi.org/10.1093/imaman/dpm022

[12] Musiela, M. and Zariphopoulou, T. (2004) An Example of Indifference Prices under Exponential Preferences. Finance and Stochastics, 8, 229-239. http://dx.doi.org/10.1007/s00780-003-0112-5

[13] Owari, K. (2010) Robust Exponential Hedging and Indifference Valuation. International Journal of Theoretical and Applied Finance, 13, 1075-1101. http://dx.doi.org/10.1142/S0219024910006121

[14] Pham, H. (2009) Continuous-time Stochastic Control and Optimization with Financial Applications. Springer-Verlag, Berlin. http://dx.doi.org/10.1007/978-3-540-89500-8

[15] Schweizer, M. (1992) Mean-Variance Hedging for General Claims. The Annals of Applied Probability, 2, 171-179. http://dx.doi.org/10.1214/aoap/1177005776

[16] Schweizer, M. (1996) Approximation Pricing and the Variance-Optimal Martingale Measure. Annals of Probability, 24, 206-236. http://dx.doi.org/10.1214/aop/1042644714

[17] Schweizer, M. (2001) A Guided Tour through Quadratic Hedging Approaches. In: Jouni, E., Cvitanic, J. and Musiela, M., Eds., Advances in Mathematical Finance, Cambridge University Press, Cambridge, 538-574. 